\title{
edoc
}

Institutional Repository of the University of Basel

University Library

Schoenbeinstrasse 18-20

CH-4056 Basel, Switzerland

http://edoc.unibas.ch/

\section{Philosophie zwischen Ursprungsdenken und Ursprungskritik}

\author{
Angehrn, Emil
}

Posted at edoc, University of Basel

Official URL: http://edoc.unibas.ch/dok/A5251720

Originally published as:

Angehrn, Emil. (2007) Philosophie zwischen Ursprungsdenken und Ursprungskritik. In: Anfang und Ursprung : die Frage nach dem Ersten in Philosophie und Kulturwissenschaft. Berlin, S. 247-274.

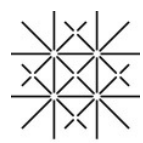




\title{
Philosophie zwischen Ursprungsdenken und Ursprungskritik
}

\author{
Emil Angehrn
}

\section{Die Frage nach dem Ursprung und ihre Kritik}

Philosophie hat mit den ersten Prinzipien zu tun. Dies ist keine beiläufige Bestimmung philosophischen Denkens. Nach dem Ursprung, dem Anfang, dem ersten Grund zu fragen, kennzeichnet die Philosophie als solche: Die reflexive Erforschung der arche definiert ihr Wesen und ihren eigenen Beginn. Wenn sich Aristoteles im Eingangskapitel der Metaphysik der, gesuchten Wissenschaft ${ }^{`}$ dadurch versichert, dass er sie in das Denkprojekt einschreibt, das seine Vorgänger verfolgten, aber noch nicht klar zu fassen vermochten, so bildet die Frage nach den Ursachen die gemeinsame Orientierung: Sie verbindet die entstehende Philosophie mit ihrer Vorgeschichte. Damit ist mehr als ein Aufweisen von Prinzipien, ein Geben von Gründen gemeint, wie es zum rationalen Argumentieren als solchem gehört. Philosophie sucht nach den ersten Gründen, nach dem radikalen Anfang und den tiefsten Wurzeln der Dinge. Nach jeder Hinsicht, unter welcher etwas begriffen werden kann, haben wir, so Aristoteles, zum ersten Prinzip zurückzugehen, wollen wir uns nicht im Unbestimmten, in der unendlichen Verkettung der Gründe verlieren und darin in Wahrheit ohne Erkenntnis bleiben. Nicht zu einem Früheren, sondern zum Ersten muss die Ursprungsbesinnung zurückgehen; nicht aus einem Vorstadium, sondern aus dem Anfang ist Wirklichkeit zu begreifen. Das Pathos des absoluten, ,voraussetzungslosen Anfangs' (arche anhypothetos) - so die prägnante (in unterschiedlichem Kontext verwendete) Formel bei Platon und Aristoteles $^{1}$ - bestimmt Anspruch und Aufgabe der Philosophie. Die Idee einer radikalen, von absoluten Prinzipien ausgehenden Erkenntnis bestimmt philosophisches Denken seit seinem Beginn und begleitet es im Ganzen seiner Geschichte.

1 Platon, Politeia 510b, 511b; Aristoteles, Metaphysik IV.3, $1005 \mathrm{~b} 14$. 
Allerdings bildet sie keine unbestrittene Leitidee des Philosophierens. Nicht nur steht sie in durchgehender Auseinandersetzung mit Positionen, die sich entweder von der Ursprungsidee als solcher oder von bestimmten Formen der Prinzipienforschung distanzieren. Darüber hinaus scheint die Ideengeschichte im Ganzen der Linie einer sukzessiven Depotenzierung des Ursprungsdenkens zu folgen, scheint sich das Denken zumal in der Moderne aus seiner anfänglichen Ursprungsverhaftung und Ursprungsorientierung herauszulösen. Während ,ehemals die Forscher, wenn sie auf dem Wege zum Ursprung der Dinge waren, immer Etwas von dem zu finden meinten, was von unschätzbarer Bedeutung für alles Handeln und Urteilen sei“, nimmt für heutiges Erkennen - so die Meinung Nietzsches - „,ie Bedeutungslosigkeit des Ursprungs $z u .{ }^{62}$ Konzepte nachmetaphysischen Denkens haben in der Kritik am Ursprungsdenken einen zentralen Kristallisationspunkt. Allerdings stellt auch dieser Wandel keinen einhelligen Sachverhalt dar: Neben Ansätzen der Kritik finden wir auch in der Philosophie des 20. Jahrhunderts - etwa in der Phänomenologie - Ansätze der Transformation und Erneuerung des Ursprungsdenkens; gegenläufig zu dessen Tot- oder Obsoleterklärung verteidigen andere das „Prestige des Ursprünglichen“ als ein „universales Phänomen, gegen das ein einsamer Entmystifizierer wie Nietzsche vergebens ankämpfen musste. “3 In Geschichte wie Gegenwart ist die Stellung des Ursprungsdenkens alles andere als eindeutig. Philosophisches Denken bewegt sich in der ungelösten Spannung zwischen Ursprungsbesinnung und Ursprungskritik.

Nun bleibt auch der so formulierte Befund unterbestimmt und seine Aussage unklar. Es bleibt unklar, worin sowohl die Bekräftigung wie die Kritik des Ursprungsdenkens besteht, und ebenso, in welcher Weise der Gegensatz zwischen ihnen Wesentliches über die philosophische Frage nach dem Ursprung auszusagen vermag. Es liegt auf der Hand, dass je nach Ausprägung des Ursprungsdenkens die Kritik an diesem anders ausfällt. Ich möchte diese Konstellation in den folgenden Überlegungen aufzuhellen versuchen und gehe dabei von einer zweifachen These aus. Sie besagt zum einen, dass die Frage nach dem Ursprung innerhalb der Philosophie durch eine grundlegende, sowohl deskriptive wie normative Ambivalenz gekennzeichnet ist. Was wir als Ursprung ins Auge

2 F. Nietzsche, Morgenröte I.44, Kritische Studienausgabe (KSA), MünchenBerlin-New York 1980, Bd. 3, S. $51 \mathrm{f}$.

3 So der Literaturwissenschaftler H. Bloom, Topographie des Fehllesens, Frankfurt am Main 1997, S. 64. 
fassen und wie wir die Frage nach dem Ursprung stellen, entfaltet sich in der Spannung zwischen entgegengesetzten Modellen und Fragerichtungen. Desgleichen oszilliert die Wertung der Ursprungsorientierung zwischen entgegengesetzten Polen, variiert das Urteil über den kognitiven Wert der Ursprungsbetrachtung, über die praktischen Motive, die ihr zugrunde liegen, und die Ziele und Bedürnisse, die wir mit ihr erfüllen. Die zweite, komplementäre These besagt, dass diese Ambivalenz nicht auszuräumen, der Antagonismus entgegengesetzter Ursprungsfiguren nicht nach der einen oder der anderen Seite aufzulösen ist, sondern dass wir uns gerade im Spannungsverhältnis divergierender Sichtweisen über die philosophische Frage nach dem Ursprung zu verständigen haben.

Ich möchte diese Gedanken in folgenden Schritten konkretisieren. Als erstes gehe ich der strukturellen, deskriptiven Differenzierung der Ursprungsfrage nach, um in einem zweiten Schritt die Antithesen zum Ursprungsdenken ins Auge zu fassen, wobei auch hier Haupttypen der Kritik - der Korrektur, Unterlaufung, Verabschiedung - des Ursprungsdenkens auseinanderzuhalten sind. Schließlich versuche ich in einem dritten Schritt beide Linien, die deskriptive und die normative Zwiespältigkeit der Ursprungsfrage, zusammenzufuihren, um sie in ihrem gegenseitigen Verhältnis und ihrer Bedeutung für die Philosophie zu reflektieren.

2. Konstellationen des Ursprungsdenkens:

Die vielfältige Frage nach dem Ersten

\subsection{Vielfalt der Ursprungsfrage}

Die Frage nach dem Grund begegnet uns in der Philosophie wie in den Wissenschaften von alters her in einer Vielfalt, die Zweifel an der Einheitlichkeit der Fragestellung aufkommen lassen kann. Sie umfasst alle Hinsichten, unter denen etwas von einem anderen her verständlich gemacht werden kann: Formal ist dasjenige, was etwas begreifen lässt, diesem gegenüber ein Früheres, eine arche, ein Prinzip. Dieses Prinzip kann von verschiedenster Art sein, es kann thematisch variieren (ein historisches Ereignis demographisch oder ökonomisch oder religionssoziologisch erklären), verschieden ,tief ${ }^{6}$ gehen (unmittelbare oder letzte Ursachen nennen), etwas nach verschiedenen Hinsichten (aus seiner Entstehung, über die herrschende Ordnung, nach Funktionszusam- 
menhängen, von bestimmten Ereignissen oder Handlungen her) intelligibel machen. Die Methodenlehre diskutiert Logik und Geltung der unterschiedlichen Weisen, einen Sachverhalt verständlich zu machen; in ihren Bereich fällt der Disput darüber, was unter welchen Bedingungen als Prinzip und Erklärungsgrund zu gelten hat.

Man kann das Spektrum der mannigfaltigen ,Gründe“ nach verschiedenen Rastern gliedern. Als Beispiel sei auf zwei älteste, aristotelische Raster verwiesen. Das eine ist die Unterscheidung, mit welcher im Begriffskatalog der Metaphysik die Begriffe der Ursache und des Grundes (arche, aitia) zusammenfassend charakterisiert werden als ,ein Erstes, von dem her etwas ist oder entsteht oder erkannt wird" ${ }^{\text {" }}$ : Die arche ist zum einen der Seinsgrund, der etwas zu dem macht, was es ist (z.B. seine Elemente oder das regulierende Gesetz), zweitens der Entstehungsgrund, aus dem etwas wird (z. B. die bewirkende Ursache oder der frühere Zustand), und drittens der Erkenntnisgrund, der etwas einsehen lässt (z.B. die Prämissen einer Argumentation). Die beiden ersten können wir mit der Differenz zwischen dem zeitlich Früheren und dem seinsmäßig Zugrundeliegenden verbinden: Der Ausgang vom Anfang und die ontologische Fundierung, die Rückkehr zur Urzeit und die Verankerung in tiefsten Gründen sind gleichermaßen in je spezifischer Weise Modalitäten des Begreifens aus der arche.

Die andere - sich teils mit der ersten überlagernde - Unterscheidung ist die traditionswirksame Vier-Ursachen-Lehre, die in zwei Begriffspaaren Hinsichten der wissenschaftlichen Erkennbarkeit auseinanderhält: ein Ding kann von seinem Stoff, seiner Wesensform, seiner Entstehungsursache und seinem Zweck her begriffen werden (in der Scholastik als causa materialis, formalis, efficiens, finalis reformuliert). Es handelt sich um theoriegeschichtlich zentrale Hinsichten, die im Blick auf die Erklärung der Naturphänomene zugleich ihren kontroversen Status erweisen: Geht es der antiken Metaphysik und Naturphilosophie darum, die Prävalenz der Formursache gegenüber dem Materialismus der Vorsokratik zu begründen, so stößt sich moderne Wissenschaft durch die Verwerfung der Finalursache zugunsten des Kausaldenkens von der Tradition ab.

Ich will mich im Folgenden nicht an diesen Unterscheidungen orientieren, sondern gleichsam quer dazu zwei andere Differenzierungen in den Vordergrund rücken, die gerade im Blick auf die Geltung der Ursachenfrage bedeutsam scheinen. Ich meine einerseits die Un-

4 Aristoteles, Metaphysik V.1, 1013a18. 
terscheidung von Anfang und Ursprung, anderseits die Differenz zwischen dem identifizierbar-erkennbaren Ersten und dem unvordenklichuneinholbaren Grund. Beides sind Unterscheidungen, die in der Diskussion um den Ursprung zum Teil thematisiert werden, zum Teil verdeckt sind und dadurch die Ursprungsfrage eigenartig schillernd werden lassen.

\subsection{Anfang und Ursprung}

Dies gilt exemplarisch für die Begriffe des Anfangs und des Ursprungs, die in unserem Problemzusammenhang vielfach als austauschbar behandelt werden. Sie werden als Übersetzungen für identische Begriffe verwendet - namentlich den Begriff der arche (während im Lateinischen die Differenz von origo und initium gegeben ist) - und bilden Referenzbegriffe in identischen Problemkonstellationen oder stehen für verwandte Figuren in einem gemeinsamen Themenbereich. Gleichwohl lässt sich ihnen - wenn wir die divergierenden Konnotationen beider Begriffe akzentuieren - ein je spezifischer Bedeutungs- und Assoziationshof, eine genuine Frage- und Interessenrichtung zuordnen. ${ }^{5}$

Der Anfang ist ein Erstes, von dem etwas ausgeht, mit dem etwas beginnt; der Ursprung ist ein Erstes, zu dem wir zurückgehen, das Späterem voraus- und zugrundeliegt. Der Anfang ist prospektiv, als Initialpunkt des von ihm Gesetzten oder aus ihm Entstehenden, der Ursprung retrospektiv, als Grund und Herkunft des Gewordenen bestimmt. Der Anfang öffnet eine Zukunft, der Ursprung weist in die Vergangenheit; im Anfangen transzendiert sich Gegenwart auf das Kommende hin, im Ursprung findet sie ihren Grund im Gewesenen. Im Rückblick bildet der Anfang einen Ausgangspunkt, der in der Vergangenheit zurücksinkt und von dem sich die Gegenwart entfernt, der Ursprung einen Wesensgrund, der im Jetzt erhalten, fundierend bleibt. Der Anfang betont das Neue und Andere, das nicht aus dem Vorausgehenden Ableitbare; der Ursprung meint das Alte, in dem Späteres wurzelt und das selbst auf Früheres zurückweisen, in Älterem gründen kann, die Kontinuität der Herkunft. Dem Pathos des Anfangens, des Anfangenkönnens, des Neubeginns auf der einen Seite steht die Sehnsucht des Ursprungs, des Zurückgehens zum Grund, des Wurzelns in der Herkunft auf der anderen gegenüber. Anfang und Ursprung markieren eine Polarität, die unter verschiedenen - tempo-

5 Den Unterschied betont M. Cacciari, Dell'Inizio, Milano 1990, S. 13. 
ralen, begründungsmäßigen, existentiellen - Aspekten artikulierbar ist. Mit beiden Begriffen sind je spezifische Probleme verbunden.

Der Anfang meint seiner basalsten Bedeutung nach den Beginn und Ausgangspunkt (einer Bewegung, einer Strecke, eines Handlungsvollzugs). ${ }^{6}$ In emphatischerem Verständnis verbindet sich damit die Vorstellung des Initialimpulses, aber auch der Neuheit und des Bruchs gegenüber dem Vorausgehenden. Davon kann in subjektiv-handlungsmäßiger wie in objektiv-ereignismäßiger Perspektive die Rede sein. Das eine Mal kommt das Anfangen und Anfangenkönnen als Spontaneität und Kreativität in den Blick : als Fähigkeit, von sich aus frei zu beginnen, als Kraft, Neues zu schaffen und die Welt originär zu gestalten. Mit Nachdruck hat Hannah Arendt in diesem Sinne das irreduzible Anfangenkönnen als Wesensmerkmal des menschlichen Seins betont. Das andere Mal geht es um ein Erleben des Neuen, das uns widerfährt, um die Unableitbarkeit des Ereignisses, das Durchbrechen des Immergleichen, das Phänomen des Plötzlichen. Im Theoretischen wie im Praktisch-Lebensweltlichen kommt dem Anfang als Handlungsvermögen wie als Ereignisform größte Bedeutung zu. In einer Welt, in der ,seinesgleichen geschieht" (R. Musil), wird das Vermögen, Neues und Anderes zu setzen und den „Bann“ (Adorno) der Wiederkehr zu brechen, zur eminenten Auszeichnung. Nicht nur die Verfassung der Welt, sondern ein Defizit der herrschenden Denkform steht zur Diskussion: Metaphysik wird als unfähig kritisiert - wie Augustinus gegen die heidnischen Philosophen hervorkehrt - , die Schöpfung der Welt und der Zeit zu denken. Allerdings bekennt er seinerseits, nicht zu wirklicher gedanklicher Klarheit über den Anfang der Zeit zu gelangen: Offensichtlich stellt das Vorhaben, den radikalen Anfang zu denken, die Theorie vor Schwierigkeiten und Aporien. Dies umso mehr, wenn die Erklärung nach gängigem Verständnis beinhaltet, etwas durch Rückführung auf anderes - eine Ursache, ein Prinzip, ein ,Früheres ${ }^{c}-\mathrm{zu}$ erklären. Anfangs- und Prinzipiendenken geraten hier in einen unmittelbaren Konflikt.

Gegenläufig zur Bewegung des Anfangens geht es beim Ursprung um Rückkehr: Das Denken geht zu einem Ersten zurück, aus dem

6 So auch die erste der sechs Bedeutungen, die Aristoteles in der Explikation der arche anführt: „Arche wird erstens derjenige Teil einer Sache genannt, von dem aus die Bewegung durch dieselbe anfängt; z. B. bei der Linie und dem Weg ist von der einen Seite dies, von der entgegengesetzten das andere Prinzip.“ (Metaphysik V.1, 1012b34-1013a1). 
etwas entsteht und in dem es seinen Grund hat. Das elementarste Schema bildet die Erzählung, die idealtypisch ein Früheres mit einem Späteren in einen Sinn- oder Funktionszusammenhang integriert, ein Späteres von einem zeitlich Vorgängigen (als Ursache, Vorbedingung, Vorstadium) her begreifbar macht. Die narrativ-zeitliche Abfolge, Grundgerüst des Mythos, wird vom rationalen Denken durch das Kausalverhältnis abgelöst: Die arche wird als eine wahrgenommen, die in irgendeiner Weise für die Existenz und Eigenart des Begründeten ,verantwortlich' ist, das ihr sein Sein und Sosein ,schuldet'. Das Woher wird durch das Woraus und Wodurch überformt, in welchem die philosophische Spekulation die Ursprungsforschung übernimmt (mit der kontroversen Anschlussfrage, ob die Materie, die Elementarteile oder die Wesensform das gründende Prinzip einer Sache seien). Spiegelbildlich zur Unableitbarkeit des Anfangs scheint auch der Fundamentalismus des Begrïndens in eine Aporie zu führen: Das Abzielen auf letzte Gründe wirft die Frage auf, wie dem unendlichen Regress oder dessen willkürlichem Abbruch zu entgehen und ein , an sich ${ }^{6}$ Erstes auszuweisen ist. Dass dies für die Prinzipienforschung kein beiläufiges Problem darstellt, wird deutlich, wenn wir die andere Hauptunterscheidung der Ursachentypen in den Blick nehmen.

\subsection{Das identifizierbare Erste und der unvordenkliche Grund}

Es handelt sich dabei um die für die begriffliche Auseinandersetzung um den Ursprung zentrale Dichotomie, die etwa als Differenz zwischen dem Grund und dem Abgrund (oder „Ungrund“) umschrieben wird: als Differenz zwischen einem Ursprung, zu dem man zurückgehen, den man freilegen, identifizieren, als gründendes Fundament sicherstellen kann, und einem Ursprung, auf den man hindenkt, der dem Zugriff entgleitet, der sich als uneinholbar vorgängiger der Benennung und klaren Erkenntnis entzieht. Der ersten Figur begegnen wir sowohl in der mythischen Reminiszenz an Gründungsereignisse, welche im Ritual als identische wiederholt werden, wie in der metaphysischen Rückführung auf substantielle Formen als Wesensgrund der Dinge oder auf einen ersten Beweger als Prinzip des Kosmos. Die zweite Figur hat ihrerseits in vielen Facetten das Denken von der Antike bis zur Gegenwart fasziniert. In ihren Bereich gehören Platons Konzept der chora, der unstrukturierten Materie und des leeren Raums, ebenso wie Plotins ,überseiendes" Eines, das die bestimmte, erkennbare Welt aus sich entlässt, und Schellings ewig dunkler Urgrund, aus dem Gott selbst erst 
zur Existenz kommt. Schellings Begriff des ,Unvordenklichen' ist zum Stichwort dieser Uneinholbarkeit des Ersten geworden; für Autoren wie Ricoeur, Derrida und Lévinas wird das unvordenkliche Vergangene - le passé immémorial - zur Chiffre der Uneinholbarkeit des Ursprungs. In kritischer Wendung wird der formlose Abgrund, der unvordenkliche Urgrund zum Gegenkonzept gegen ein ,identifizierendes' Ursprungsdenken. Strukturell wird das Unbestimmte als das dem Bestimmten Vorausliegende zum Grund erklärt. Temporal geht es um einen Vergangenheitsbezug, der sich nicht am Damals eines Urgeschehens, sondern am Je-schon eines Vorgängigen ausrichtet; ein Leitbegriff, unter dem diese Figur in neueren Debatten (so bei Derrida und Lévinas) evoziert wird, ist der Begriff der Spur - der Spur, welche ein Anderes hinterlassen hat, das nicht mehr gegenwärtig und in Präsenz zu überführen ist.

In idealtypischer Gestalt wird die Opposition dieser beiden Ursprungsfiguren am Beginn unserer Denkgeschichte fassbar. Wenn üblicherweise zwar die entstehende Metaphysik gerade in ihrer Suche nach der arche als Nachfolgerin des Mythos gilt, so überdeckt diese Kontinuität eine ebenso tiefe Divergenz. Was der Mythos als ältesten Ursprung vor Augen stellt und was die Metaphysik als Prinzip herausarbeitet, sind antagonistische Instanzen des Ersten. Der Schöpfungsmythos berichtet vom Heraufkommen der Welt aus dem Ungeordnet-Ungeformten. Die erste Macht, mit der in Hesiods Theogonie die Entstehung der Götter und Menschen einsetzt, heißt Chaos; das Tohuwabohu (,wüst und leer ${ }^{6}$ ) der biblischen Schöpfungserzählung, das Unbestimmt-Unbegrenzte (apeiron), aus welchem bei Anaximander die Gegensätze und Welten hervorkommen, sind Figurationen dieses Ungestalten, das der bestimmten Form und strukturierten Welt vorausliegt. Es liegt dem voraus, was für die Metaphysik allein Realprinzip wie Erkenntnisgrund sein kann. Die platonische Idee, die aristotelische substantielle Form stehen paradigmatisch für dasjenige, was durch sich selbst erkennbar und an ihm selbst existierend ist und was darin zugleich den Grund des Seins und Erkennens von anderem bildet. Es hat diese privilegierte Stellung dadurch, dass es das absolut Bestimmte, das mit sich Identische, Aktuale und Ungemischte - ohne Zonen der Diffusität, Potentialität, Instabilität und Wandelbarkeit - ist. Demgegenüber gelangt das Erkennen im Maße des Zurückgehens zu tieferen, älteren Fundamenten nicht zu einer höheren Stufe, sondern verliert sich im Unbestimmten und Dunklen. Die chora, die als kosmischer Urstoff aller Ordnung und Gestalt vorausliegt, ,entflieht" nach Platon der Identifizierung und ist 
begrifflich, ,äußerst schwierig zu fassen“.7 Führt der Rückgang zum ersten Prinzip im einen Fall zu einem in eminenter Weise Erkennbaren, so im anderen zu einem Jenseits von Sprache und Wissen.

Die Opposition von solcherart divergierenden Ursprungsbezügen findet sich in analoger Gestalt in variierenden Konstellationen der Ideengeschichte. Sie kristallisiert sich in verwandten logisch-ontologischen Gegensätzen: Dem Streit um die ontologische Priorität zwischen Stoff und Form, Potenz und Akt, Differenz und Identität, Unendlichem und Bestimmtem korrespondiert die Auseinandersetzung um den wahren Ursprung aller Dinge. Sie ist auch in nicht-spekulativen $\mathrm{Zu}-$ sammenhängen als Alternative durchaus fassbar. $\mathrm{Ob}$ wir eine soziale Institution eher von ihrem bestimmten Gründungsakt oder der weiter zurückreichenden, komplexen und im Dunkel sich verlierenden Vorgeschichte her begreifen sollen, ob wir einen Rechtstext vom Wortlaut des Gesetzes oder von seiner Genese und seinem impliziten Subtext, auch dem Ausgeschlossenen und Nichtgesagten her auslegen sollen dies sind nicht abstrakt-konzeptionelle Optionen, sondern offene Fragen, welche sich im Alltag wie in der Wissenschaft stellen und die Entscheidung zwischen ganz unterschiedlichen Weisen, etwas von seinem Grund her zu verstehen, betreffen. Dabei kann die Figur des entgleitenden, uneinholbaren Grundes ihrerseits in schillernder Gestalt vorkommen, sofern sie einerseits auf das Diesseits, anderseits das Jenseits der intelligiblen Gestalt verweist. Sowohl das abgründige Chaos wie das mystische All-Eine entziehen sich der identifizierenden Benennung; die reine Nacht und die Überfülle des Lichts können sich spiegeln, der anfänglichste Urgrund und die jenseitige Vollendung in vergleichbaren Bildern als Innerstes der Dinge fungieren.

All diese Figuren machen deutlich, dass ein einheitliches Urteil über das Verhältnis von Ursprungsorientierung und Ursprungskritik schon dadurch erschwert wird, dass die Ursprungsfrage selbst in ganz verschiedener, teils antithetischer Prägung auftritt. In welcher Weise die strukturelle Heterogenität und die normativ-wertende Ambivalenz der Ursprungsfrage zusammenspielen, bleibt zu präzisieren.

7 Platon, Timaios $49 \mathrm{e}, 51 \mathrm{a}-\mathrm{b}$. 


\section{Kritik und Radikalisierung des Ursprungsdenkens}

Die typologische Differenz der Ursprungsbetrachtung konfrontiert uns nicht einfach mit einem Spektrum von Varianten, sondern mit einer Kontroverse, die sich gleichzeitig als Kritik - an bestimmten Ursprungsfiguren oder am Ursprungsdenken als solchem - artikuliert. Aus den vielfältigen Linien dieser Kontroverse seien drei Frontstellungen herausgehoben, die für die Ursprungsproblematik von exemplarischer Bedeutung sind. Sie betreffen einerseits die metaphysische Gegenwendung zur mythischen Ursprungsbesinnung (3.1), anderseits die Kritik am metaphysischen Prinzipiendenken, welches teils zurückgewiesen und verabschiedet (3.2), teils transformiert und radikalisiert (3.3) wird.

\subsection{Metaphysische vs. mythische arche:}

Die Überwindung des Chaos durch Ordnung und Gestalt

An die Stelle des dunklen Urgrundes setzt Metaphysik den festen Grund. Unverkennbar behauptet sich der Fundamentalismus der Metaphysik in einer Bewegung der Abwehr - gegen die zerstörerischen Mächte des Chaos, die ungeordnete Vielfalt, die Diffusion zwischen Wesentlichem und Unwesentlichem, Formlosigkeit und Gestalt. Diese Frontstellung manifestiert sich im Herzstück der metaphysica generalis wie der metaphysica specialis; sie begegnet uns beidemal bei Aristoteles in profilierter Weise, wobei das eine Mal die vorsokratische Naturphilosophie, das andere Mal zusätzlich der Mythos die Gegenposition bildet. Im ersten Fall geht es um den Nachweis, dass dasjenige, was das Wesen der Dinge ausmacht, nicht der Stoff oder das Substrat sein kann, da nur die Form (Essenz) der Sache eindeutige Bestimmtheit verleiht und damit auch ihre Individualität und ihre Existenz begründet. Nicht die Materie (Holz), sondern die Speziesbestimmung (Kirschbaum) macht einen Gegenstand zum individuierten, aktual existierenden Ding; nur durch den Bezug auf das eidos gewinnt die Sprache Konsistenz und Erkenntniskraft. ${ }^{8}$ Dies ist die essentialistische Grundthese klassischer Metaphysik. Im zweiten Fall geht es darum, gegen das Hervorkommen des Kosmos aus Chaos und Nacht die ewige Ordnung der Welt zu beweisen: Dazu muss auf eine immer tätige Ursache zurückgegangen werden, in welcher keine Potentialität und kein Stoff (als Dimension des Potentiellen) vorhanden sein können. Wäre die dynamis, nicht die en-

8 Aristoteles, Metaphysik VII.4-6. 
ergeia das Frühere, „so würde nichts von dem Seienden sein“: Die „alte Götterlehre, welche alles aus der Nacht erzeugt", vermag die Genese und Ordnung der Welt ebensowenig zu erklären, wie die Ansicht der Naturphilosophen, dass am Anfang ,alle Dinge beisammen waren“.

In beiden Fällen handelt es sich um die Zurückweisung einer bestimmten Erklärungslogik, die das Höhere aus dem Niedrigeren, das Sein aus dem (Noch-)Nichtsein, die Gestalt aus der Formlosigkeit, die aktuale Bewegung aus der Potenz herzuleiten versucht. Es ist die Kritik an einer bestimmten Ursprungslogik, die im Rückgang zu den Prinzipien gleichsam ,fundamentalistischer, radikaler als die Metaphysik ansetzen und hinter die bestehende Welt und die identifizierbaren Dinge zurückgehen will. In gewissem Sinn geht es hier um einen sowohl ontologischen wie erklärungslogischen Disput und zugleich einen Rechtsstreit in der Verwendung des Begriffs: Die dezidierte Forderung, zu den ersten Prinzipien zurückzugehen ${ }^{10}$, verbindet sich mit der These, dass nur das in sich Intelligible, d. h. das Begrenzt-Bestimmte, nicht das Unendliche ein solcher Ursprung sein kann. Zurückgewiesen wird die Auffassung, dass die materialistische bzw. mythologische arche der wahre, eigentliche Ursprung sei. Nur scheinbar ist die Herleitung aus dem Chaos radikaler als die Rückführung auf das dem Kosmos immanente Bewegungs- und Ordnungsprinzip.

Indessen handelt es sich nicht allein um einen logischen Disput. Dieser selbst bleibt bekanntlich im Lauf der Ideengeschichte offen; Metaphysikkritik begleitet die Metaphysik wie ein Schatten und fuihrt periodisch zur Umkehrung der statuierten Rangordnung zwischen Wesen und Akzidens, Identität und Differenz, Substanz und Funktion. $\mathrm{Ob}$ wir etwas in der Fokussierung auf seinen Wesenskern oder in der Entfaltung seiner relationalen Bezüge adäquater erfassen, wird nicht ein für allemal zwingend entschieden. Wichtig sind die Intuitionen und Motive, welche der einen oder der anderen Stellungnahme zugrundeliegen. Diese Motive, die in den Weltentstehungsmythen in deutlichster Gestalt hervortreten, finden bezeichnenderweise auch in der entstehenden Metaphysik ihren Niederschlag. In typischen Schöpfungsmythen wird nicht ein harmonisches Hervorgehen aus dem Urgrund, sondern ein kosmischer Urkonflikt geschildert, in welchem die Mächte der Zerstörung am Ende durch die Mächte des Seins überwältigt werden. Ausgetragen wird er im Generationenkonflikt zwischen alten

9 Aristoteles, Metaphysik XII.6, 1071b24-28.

10 Aristoteles, Metaphysik, II.2. 
und neuen Göttern, im wiederholten Kampf gegen die als Drachen, Schlangen, Seeungeheuer auftretenden Mächte der Unterwelt. In drastischen Schilderungen evoziert die Theogonie den Schrecken vor der urtümlichen Verwirrung und Grenzenlosigkeit. Einen Reflex der ursprünglichen Angst vor der Konfusion der Formen und dem UnfassbarUnendlichen können wir ebenso in der entstehenden Metaphysik wahrnehmen. Die von Parmenides auseinandergehaltenen Wege des Denkens mit der scharfen Verurteilung der Unentschiedenen, die nicht zwischen den Wegen des Seins und des Nichtseins unterscheiden können, die Beschreibung des wahrhaften Seins als eines in jeder Hinsicht mit sich Identischen, das keiner Mischung und keinem Wandel unterworfen ist, das Bemühen um die Reinheit der Form und die Transparenz der Ordnung sind analoge Formen der Abwehr jeder Art von Kontamination, Unklarheit und grenzüberschreitender Diffusion.

Es sind Strategien eines Strebens nach Sicherheit, das Halt am Festen und Bestimmten sucht, Formen der Bewältigung einer ursprünglichen Angst, die im Tiefsten nicht eine Angst vor der fremden Übermacht, sondern - wie von der modernen Existenzphilosophie bestätigt - vor dem Unbestimmten und nicht Festlegbaren, vor dem Abgrund, dem Stürzen ins Leere ist. Metaphern dieses Affekts und dieses Strebens finden wir im Durchgang durch die Denkgeschichte, angefangen bei Platon und Aristoteles, der beispielsweise das Prinzip des ausgeschlossenen Widerspruchs, welches den ,voraussetzungslosen Anfang' bildet, auf 15 Zeilen fünfmal als das ,sicherste Prinzip“ von allen beschreibt. ${ }^{11}$ Nicht weniger aussagekräftig ist die Beschreibung des Ersten Bewegers, der durch seine immanente Notwendigkeit als reiner Akt die Regularität der kosmischen Bewegung verbürgt - so dass, wie Aristoteles anfügt, ,die Sonne, die Gestirne und der ganze Himmel stets in Bewegung sind und man keine Angst zu haben braucht, dass sie einmal still stehen, wie dies die Naturphilosophen befürchten “12. Die scheinbar nebensächliche Bemerkung zur Angst der Naturphilosophen ist Indiz für ein treibendes Motiv metaphysischer Ursprungssuche, die auf Selbstvergewisserung und Angstbewältigung zielt. Das fundamentum inconcussum, das Descartes im Cogito findet, wird hier an der unerschütterlichen Festigkeit des ersten Grundes festgemacht. Sich auf diesen festzulegen heißt zugleich ein noch Tieferes, Abgründiges abzuwehren: Metaphysik ist Kritik mythischer Ursprungsbesinnung. Es liegt nahe, dieses Dispositiv

11 Aristoteles, Metaphysik IV.3, 1005b9-23.

12 Aristoteles, Metaphysik IX.8, 1050b22-24. 
seinerseits kritisch zu lesen: die Zurückweisung als Verdrängung einer Negativität zu deuten, die in der Tiefe der Lebenswelt bestehen bleibt und durch die Ordnung eingedämmt, doch nie endgültig eliminiert wird. Dieser Negativität ist sich der Mythos bewusst, wenn er sie in der rituellen Wiederholung vergegenwärtigt und sich je neu mit ihr auseinandersetzt. Metaphysische Ursprungsforschung wäre nach dieser Lesart gerade in der Frontstellung gegen den anderen, älteren Ursprung ein Verfahren des Ausschlusses und der Unterdrückung. Ein Strang der neueren Metaphysikkritik, der auf die Verengung okzidentaler Vernunftkultur und die Marginalisierung von Pluralität, Differenz und Kontingenz abhebt, lässt sich in Affinität zu diesem Vorbehalt verstehen.

\subsection{Die Kritik am metaphysischen Fundamentalismus}

Damit kommt die zweite Konstellation der Ursprungskritik in den Blick: die Konstellation jener Kritik, die sich gegen die metaphysische Prinzipienforschung richtet. Sie tut dies zum einen so, dass sie die Orientierung an der Ursprungsfrage als solche kritisiert. Einen prominenten Einsatzpunkt in der Moderne stellt die eingangs genannte Kritik Nietzsches an der Hypostasierung des Ursprungs dar. Für ihn selbst wird damit nicht die Erforschung der Herkunft disqualifiziert: Vielmehr wird - gegen das ,wesentlich unhistorische“ Denken der Philosophie - eine historisch-genealogische Betrachtung gefordert, die sich jedoch von der teleologischen und linear-vereinheitlichenden Sichtweise der Geschichtsphilosophie freimacht und namentlich die metaphysische Ebenendifferenz zwischen Ursprung und Derivat verwirft. ${ }^{13}$ Exemplarisch geht es im Bereich der Moral, Religion und Kunst darum, anstelle der Herleitung aus höheren Prinzipien - z. B. einem kantischen Sittengesetz - die reale, niedrige Herkunft (aus Affekten und ,abscheulichen kleinen Schlüssen“ - „o pudenda origo!“) aufzuweisen. ${ }^{14}$ Solche Umdeutung bedeutet einen Affront für das normale Moralempfinden oder ästhetische Bewusstsein. Ähnlich verbindet M. Foucault die normative wie ontologische Depotenzierung des Ursprungs mit einer revidierten historischen Betrachtung. Explizit auf dem Spiel steht die metaphysische Verfassung der zurückgewiesenen Ursprungsforschung: Anstelle des notwendigen, wesentlichen, identischen Grundes gilt es die zufälligen,

13 F. Nietzsche, Menschliches, Allzumenschliches I.1, KSA, Bd. 2, S. 23 f.; vgl. Zur Genealogie der Moral, I.2, KSA, Bd. 5, S. $254 \mathrm{ff}$.

14 F. Nietzsche, Morgenröte, II. 102, KSA, Bd. 3, S. 90. 
vielfältigen und zerstreuten Anfänge aufzuzeigen, anstelle der sinnhaften Entwicklungsgeschichte geht es um die Archäologie der Materialien, statt der verbindenden Kontinuität um die Kontingenzen und Brüche. Damit verändert sich die Funktion des Rückgangs zum Grund: Statt ein Gewordenes in seiner Identität zu befestigen und seine Geltung zu legitimieren, fuihrt Genealogie zur kritischen Befragung und zur Zersetzung der einheitlichen Gestalt. Sie sucht ein historisches Verständnis ohne Einswerden mit der Urzeit und Rückbeziehung auf ein letztes Fundament. Sie vollzieht eine dezidierte Gegenwendung gegen das, was im prägnanten Sinn Ursprungsforschung heißen kann.

Zum eigentlichen Topos wird die Kritik am Ursprungsdenken bei Th. W. Adorno. Sie ist umfassend angelegt und schlägt den Bogen von der konzeptuellen Prägung der Metaphysik über den Fundamentalismus der phänomenologischen Beschreibung bis zur Blut-und-Boden-Ideologie des Nationalsozialismus. Gegen den Ausgang von einem „schlechterdings Ersten überhaupt ${ }^{\text {"15 }}$ teilt Negative Dialektik die Hegelsche These von der unhintergehbaren Vermitteltheit aller Dinge: Es gibt nicht den irreduziblen Ausgangs- und Gründungspunkt, weder in der Erkenntnis noch im Sein. ${ }^{16}$ Epistemologisch liegt darin die Kritik am unmittelbaren Wissen: Was Hegel in der Phänomenologie des Geistes für die sinnliche Gewissheit durchführt, moniert Adorno in Husserls Ansatz bei einer "Seinssphäre absoluter Ursprünge" ${ }^{\text {17 }}$ (oder analog in Bergsons „données immédiates de la conscience ${ }^{\text {"18 }}$ ). Ontologisch gilt die Kritik dem metaphysischen Fundamentalismus, der im Ursprung nicht nur das Frühere, sondern das Wesentliche zu ergreifen meint. ${ }^{19}$ In Wahrheit ist das, was aller Vermittlung voraus- und zugrundeliegt, nicht das wahre Wesen und sichere Fundament, sondern ein abstraktes Residuum nach Ausschaltung aller vermittelten Bestimmtheit, „ganz dürftig und leer". ${ }^{20}$

Zugleich erkennt Adorno in der metaphysischen Ursprungssuche mehr als eine konzeptuelle Verfehlung. Er sieht in ihr eine Suche nach

15 Th. W. Adorno, Metaphysik. Begriff und Probleme, Frankfurt am Main 1998, S. 68.

16 Ebd. S. 48 f.; vgl. S. 15, 25, 70 f., 216 f.

17 Th. W. Adorno, Zur Metakritik der Erkenntnistheorie, in: Gesammelte Schriften, Bd. 5., S. 12.

18 Vgl. ebd. S.49.

19 Th. W. Adorno, Metaphysik, S. 224 f.; Negative Dialektik, Frankfurt am Main 1966, S. $140 \mathrm{ff}$.

20 Th. W. Adorno, Zur Metakritik der Erkenntnistheorie, S. 15, 23. 
dem Festgelegten und Stabilen, in welcher ein abgründiges Sicherheitsbedürfnis zum Ausdruck kommt, das seinerseits die metaphysische Fragerichtung prägt. Während Dialektik mit dem Bodenlosen, Schwindelerregenden, Zerbrechlichen konfrontiert und darin jenem Halt entsagt, den die Suche nach dem Ersten verspricht, ist Ursprungsphilosophie von einem Vorurteil für das Einfache und Primitive getragen und durch eine „Tendenz zur Regression“ bestimmt. ${ }^{21}$ Die im arche-Begriff seit Aristoteles immer wieder herausgestellte Doppelbedeutung von Anfang und Herrschaft wird als nicht-kontingente Verbindung reflektiert: als Herrschaftlichkeit der Älteren und früher Dagewesenen, genereller als Zeichen der Vorherrschaft der Vergangenheit über die Zukunft, als Zwang der Reproduktion des Gleichen und Ausschluss des Neuen. Nicht zuletzt bringt Adorno das Fiktive, aber auch Unheilvolle der Ursprungsnähe in ideologiekritischer Perspektive innerhalb des philosophischen Diskurses zur Sprache, wobei als privilegierter Adressat Heidegger dient, in dessen Werk nach Adorno plastisch fassbar wird, wie die fundamentalontologische Beschwörung des Ersten sich ob ihrer Leere dem Allerrelativsten anheimgibt: Die Berufung auf das Bäuerliche und Ursprüngliche wird zur Komplementärideologie innerhalb einer industrialisiert-kapitalistischen Welt, der sie sich in Wahrheit nicht als Anderes entgegenstellt, sondern reibungslos einpasst. $^{22}$ Insofern ist die Kritik am Ursprungsdenken in gewisser Hinsicht auch eine an der Usurpierung des Begriffs, doch ohne dass ein anderer, wahrerer Ursprung an Stelle des leeren Anspruchs gesetzt werden soll. Vielmehr ist in der kritisierten Haltung eine Tendenz zur schlechten Ursprünglichkeit auszumachen, der sich ein kritisches Denken ebenso zu widersetzen hat wie den ontologisch-epistemologischen Hypostasierungen. Auch andere Autoren haben die ,ursprungsmythische' Haltung in politischen und intellektuellen Zeitströmungen diagnostiziert und die Ursprungsorientierung im Zeichen der Repression und Reaktion gedeutet. ${ }^{23}$

Wenn Ursprungskritik in solchen Konzepten ihre Dringlichkeit dadurch gewinnt, dass sie über die theoretische Denkform hinausgreift

21 Ebd. S. 27.

22 Vgl. Th. W. Adorno, Jargon der Eigentlichkeit, Frankfurt am Main 1964; Philosophische Terminologie, Frankfurt am Main 1973, Bd. 1, Vorl. 13 u. 14.

23 Für P. Tillich ist das ,ursprungsmythische Bewusstsein [...] die Wurzel alles konservativen und romantischen Denkens in der Politik": Die sozialistische Entscheidung [1933], Berlin 1980, S. 18. Vgl. K. Heinrich, Parmenides und Iona, Basel / Frankfurt am Main 1982. 
und zugleich geschichtlich-politische Bezüge ins Spiel bringt, so zielen andere vorrangig auf die Überwindung der zugrunde liegenden psychischen und intellektuellen Haltung. Ihnen geht es darum, von einem falschen Bedürfnis freizukommen, oder positiv: mit jenem Gefühl des Schwindelerregenden und Haltlosen, auf welches der metaphysische Fundamentalismus reagiert, zurechtzukommen. Die Forderung ist, sich vom gründenden Prinzip und sicheren Boden ohne Nostalgie zu verabschieden: Das Denken soll ohne die Zentrierung auf Einheit, Ganzheit und Notwendigkeit auskommen. Als Beispiele sei auf R. Rortys Plädoyer für Kontingenz oder O. Marquards Apologie des Zufälligen und seine Rehabilitierung der Pluralität verwiesen ${ }^{24}$; in pointierter Form hat N. Luhmann den Verzicht auf die ,alteuropäische“ Orientierung am Wesentlichen zum Leitmotiv der Theorie gemacht. Mit einer sprechenden Formulierung hat Botho Strauß diesen Wandel mit der Ursprungsfrage verknüpft und die postulierte Einstellungsänderung in die Figur der „Beginnlosigkeit“ eingezeichnet. ${ }^{25}$ Gemeint ist eine Haltung, die sich im Offenen und Komplexen $\mathrm{zu}$ orientieren vermag und sich vom Bedürfnis freigemacht hat, das Komplexe durch Rückfuihrung auf seine einfachen Elemente zu durchschauen und zu beherrschen. Es ist das Bewusstsein, immer schon mitten drin, unterwegs zu sein und die Sicherheit, ja Selbstverständlichkeit der Orientierung ohne „Begradigung' der Welt gemäß einer linearen Verkettung im Ausgang von ersten Prinzipien zu gewinnen. Es ist gewissermaßen ein Plädoyer für die Leichtigkeit des Seins, ein Plädoyer dafür, sich nicht im Ursprung, sondern im Nichtursprünglichen, in dem, was ,später ${ }^{6}$ als der Ursprung ist, einzurichten und auszukennen. In dieser Linie verliert die Kritik am Ursprungsdenken etwas von dem denunziatorischen Ton und der Dramatik, die ihr in ideologiekritischen Kontexten zukommt: Sie verweist auf den Konstruktcharakter der Ursprungsbilder und möchte sich damit begnügen, die Fixierung auf diese als obsolet und lebenshinderlich zurückzulassen. Allerdings mag auch dieser Gestus nicht so problemlos sein, wie er sich gibt. Luhmanns obstinater Appell an das Aushaltenkönnen der Kontingenz ${ }^{26}$ ist nicht ohne ein Pathos, das etwas von der Schwierigkeit der Zumutung erkennen lässt. Womöglich

24 Vgl. R. Rorty, Kontingenz, Ironie und Solidarität, Frankfurt am Main 1989; O. Marquard, Apologie des Zufälligen. Philosophische Studien, Stuttgart 1986.

25 B. Strauß, Beginnlosigkeit. Über Fleck und Linie, München / Wien 1992.

26 Vgl. N. Luhmann, Politische Theorie im Wohlfahrtsstaat, München 1981, S. 23, 125. 
kann sich die Ursprungskritik, die das Ursprungsdenken ohne Rest liquidieren, es als falsches Prinzip abschreiben will, doch nicht so umstandslos von ihm freimachen, wie es ihrer Absicht entspricht.

\subsection{Ursprünglicher als der Ursprung}

Dieser Schwierigkeit entgeht jene andere Kritik, die das Ursprungsdenken nicht nur auflösen, sondern gleichsam hinter es zurückgehen, es unterlaufen und vertiefen will. Auch dieser Ansatz ist mit einer dezidierten Kritik an der metaphysischen arche verbunden, doch bleibt in gewisser Schwebe, wieweit er im Ganzen eher als transformiertes Ursprungsdenken oder als Ursprungskritik zu fassen ist. Klar ist, dass auch dort, wo ein Früheres, Ursprünglicheres als der Ursprung ins Auge gefasst ist, diese Radikalisierung nicht einfach bedeutet, einen Schritt weiterzugehen und anstelle des ersten, konstruierten, einen anderen, authentischeren Ursprung auszumachen. Vielmehr geht es darum, einen Ursprung anderer Art, etwas anderes als den - metaphysisch oder mythisch definierten - Ursprung zu denken. Offenkundig ist die hier anvisierte Differenz mit jener verwandt, die zwischen den idealtypischen Figuren des identifizierbaren Ersten und des unvordenklichen Grundes sichtbar geworden ist. Diese Gegenwendung lässt sich exemplarisch anhand von zwei Figuren verdeutlichen, deren zweite sich selbst kritisch gegen die erste wendet: Heideggers Konzept der Seinsgeschichte und Derridas Begriff der différance.

Heideggers grundlegende Kritik an der philosophischen Tradition seit Platon ist wesentlich eine Kritik an der herrschenden Sichtweise vom Ersten und vom Fundament. Das Stichwort der Seinsvergessenheit, mit dem Heidegger das Defizit der abendländischen Metaphysik benennt, meint eine Ursprungsvergessenheit: Metaphysik, die sich zwar ihrem aristotelischen Begriff nach über die Suche nach den ersten Prinzipien definiert, denkt gerade nicht vom Ersten, vom Anfang her. Die Seinsvergessenheit besteht darin, innerhalb der von Heidegger herausgestellten ,ontologischen Differenz' zwischen Sein und Seiendem das Ursprüngliche zugunsten des Abgeleiteten zu unterschlagen. Ginge es in Wahrheit darum, das Seinsgeschehen als ein Offenbarungsgeschehen, ein Zur-Erscheinung-Kommen der. Dinge zu denken und darin gleichsam die Konstitution der Wirklichkeit aus ihrem Ursprung nachzuvollziehen, so geht Metaphysik vom konstitutierten Gegenstand, vom Seienden als Seienden aus, das nicht in seinem Hervorgehen, sondern in seinem fertigen Konstituiertsein betrachtet wird. Das Projekt 
einer Überwindung der Metaphysik wäre nichts anderes als ein Hinauskommen über diese ursprüngliche Vergessenheit des Seins ${ }^{27}$ - eine Wiedergewinnung der ,Anfänglichkeit ${ }^{\star}$ durch ein Denken, das in kritischer Auseinandersetzung mit dem ersten, verhüllenden Anfang den „anderen" Anfang vorbereitet, in welchem das Sein als Ereignis und Grund des Seienden erfahrbar wird. ${ }^{28}$ Allerdings ist das Heraufkommen dieses anderen Anfangs nach Heidegger ein Geschehen, das selbst nicht in der Hand des Subjekts liegt, sondern sich als ,objektives' Ereignis in der Geschichte des Seins vollzieht.

Die Frage, wie mit dem Ursprung umzugehen sei, betrifft Derridas Philosophieren in seinem Kern und im Ganzen. Allgegenwärtig ist nicht nur die kritische Distanzierung, sondern ebenso die Tendenz zur Überbietung der klassischen Prinzipienforschung, die in eigensinnigen Begriffswendungen zum Ausdruck kommt: so wenn der Begriff der ,Spur' für etwas eingesetzt wird, was ,, ursprünglicher ${ }^{6}$ als die phänomenologische Ursprünglichkeit“"29, gleichsam „Ursprung des Ursprungs "30 sein soll, oder wenn die Dekonstruktion mit der psychoanalytischen Suche nach einem „Ur-Ursprünglichen“ bzw. einem „archi-originaire plus , ancien“ que l'origine“"31 verglichen wird, nicht zuletzt in den zu Leitbegriffen erhobenen Composita mit dem Präfix ,archi-' wie in der Ur-Schrift und Ur-Spur (archi-écriture, archi-trace). Gerade der Begriff der Spur insistiert darauf, dass wir nie vom Nullpunkt anfangen, sondern in unserem Verstehen wie im Sprechen und Handeln immer schon unterwegs sind. Der Begriff steht exemplarisch für jene Umkehrung des metaphysischen Ursprungsdenkens, die nicht ein Anderes an der Ursprungsstelle einsetzen will - das Viele anstelle des Einen, die Relation anstelle der Substanz -, sondern den Gedanken des Ursprungs als eines identifizierbaren Ersten als solchen unterminiert. Die Spur als ursprünglich anzusehen bedeutet nicht nur, dass wir nie Zeitgenossen des Anfangs sind und das schlechthin Anfängliche festhalten können, sondern dass in einem radikalen Sinn kein absoluter Anfang ist. Ursprünglich ist nicht das Element, aus dem sich Komplexes

27 M. Heidegger, „Überwindung der Metaphysik“, in: Vorträge und Aufsätze, Pfullingen 1954, S. 67-96, S. 74; „Einleitung zu: ,Was ist Metaphysik?““, in: Vorträge und Aufsätze, a. a. O., S. 198.

28 M. Heidegger, Beiträge zur Philosophie (Vom Ereignis), in: Gesamtausgabe I, Bd. 65, S. 31.

29 J. Derrida, La voix et le phénomène, Paris 1967, S. 75.

30 J. Derrida, De la grammatologie, Paris 1967, S. 90.

31 J. Derrida, Résistances à la psychanalyse, Paris 1996, S. 41, 44. 
zusammensetzt, sondern die „complication originaire“32, die je schon gegebene Verwicklung des Anfänglichen mit dem aus ihm Kommenden, die unhintergehbare Kontamination des Ersten durch das Zweite, wobei für Derrida die Irritation der Ursprünglichkeit des Nichtursprünglichen erhalten bleibt: „Comment tout peut-il commencer par une complication?" ${ }^{\text {33 }}$ In der Zurückweisung des Ersten bleibt die Ursprungsfrage virulent. Wie Derrida darauf insistiert, dass die différance, der immer erneute Aufschub des Einswerdens, ohne den spannungsgeladenen Bezug zur Identität sich gleichsam verflüchtigt, so bleibt auch im Entgleiten des Ersten der Bezug auf den Ursprung nicht eliminierbar.

Verschiedene Figuren dienen Derrida dazu, dieses Ineinander von Entzug des Ursprungs und Ursprungsbezug zu umschreiben. Wenn die ursprüngliche ,Kontamination“ gewissermaßen den Rückschlag des Zweiten auf das Erste, die Nicht-Absolutheit des Anfangs meint, so betont die von Husserl übernommene Figur der ,Iteration' den Vorverweis des Ersten auf das Zweite. Jede Setzung ist eine, die auf ihre Wiederaufnahme und Kritik vorausweist: Alles ist, was es gewesen sein wird. Innerhalb des eigensten Terrains der Dekonstruktion geht Derrida dieser Struktur anhand der Figuren der Lektüre und des Schreibens nach: Lektüre geht nicht auf das feststehende Original als Urtext, sondern auf eine ,Urschrift' als ursprüngliche Einschreibung zurück, die dem Geschriebenen noch vorausliegt. Die Sinngenese ist auf der einen Seite ohne ersten Initialpunkt, auf der anderen unabgeschlossen und immer schon auf ihre Wiederholung und Transformation ausgreifend. Das Zweite - zweite Mal - überlagert je schon das Erste: „C'est l'idée même de première fois qui devient énigmatique. “34 Die Spur wird zur Chiffre dieses anders gefassten, weder in Nichts aufgelösten noch je in Präsenz überfuihrbaren Ursprungs. Dekonstruktive Lektüre, die den Text überschreibt, ist eine Übersetzung ohne Original; Gegenwart wurzelt in einem „Vergangenen, das nie gegenwärtig war. ${ }^{635}$ Die Ursprungslosigkeit ist als das eigentliche Ursprüngliche anzuerkennen:

32 J. Derrida, Le problème de la genèse dans la philosophie de Husserl, Paris 1990, VI-VII; Résistances à la psychanalyse, S. 48.

33 Ebd., VII, S. 12.

34 J. Derrida, L'écriture et la différence, Paris ${ }^{2} 1979$, S. 301; vgl. Gesetzeskraft, Frankfurt am Main 1991, S. 83 f.

35 M. Merleau-Ponty, Phénoménologie de la perception, Paris 1945, S. 280; J. Derrida, „La différance“, in: Marges de la philosophie, Paris 1972, S. 22 (mit Bezug auf Lévinas). 
„C'est la non-origine qui est originaire. “36 Es ist ein Zurückgreifen hinter den Ursprung, das in diesem Sinn auf ein Älteres, ,Ursprünglicheres $^{637}$ geht, doch ohne dass dieses nun zur wahren arche mutierte: Hinter dem Ursprung liegt nicht ein wahrerer, eigentlicher Ursprung. Nicht zuletzt kennzeichnet Derrida das Genuine dieser ,Subversion' des Ursprungsdenkens in Antithese zu Heidegger, in dessen Gedanken des ,Seins" er gerade eine Neubesetzung der Ursprungsstelle sieht, ein Zurückgehen in der Kette der Ursprünge zu einem noch Ursprünglicheren als die Dinge und Substanzen, für welches am Ende doch ein Eigenname, ein einziger Name stünde. Die Preisgabe der Hoffnung auf den endlich zu findenden, eigentlichen Namen definiert die von Derrida intendierte Umkehrung des Denkens. Die différance, ,,älter a als das Sein selbst, hat keinen Namen in unserer Sprache": sie ist nicht selbst als Ursprung identifizierbar, sondern dasjenige, auf welches wir hindenken als Herkunft jeder Bestimmtheit und jeden Namens. ${ }^{38}$

\section{Die Ambivalenz des Ursprungs und die Philosophie}

Nicht nur die Suche nach dem Ursprung, auch der Antagonismus zwischen Ursprungsdenken und Ursprungskritik lässt sich nicht auf eine Formel reduzieren. Wenn wir die strukturelle Heterogenität und die antithetische Wertung der Ursprungsfigur miteinander verflechten, können wir folgende Konstellationen festhalten.

\subsection{Die zweifache Ursprungskritik}

Ausgangspunkt ist der zweifache Ansatz der Kritik, genauer: die Dialektik von Kritik und Gegenkritik: einerseits die ,metaphysische' Kritik am mythischen Ursprungsbezug, anderseits die nachmetaphysische Kritik an metaphysischen Ursprungsfiguren. Auf der einen Seite haben wir den Einspruch gegen die Herleitung der Welt aus dem Chaos, gegen die Begründung des Höheren aus dem Niedrigeren, der Form aus der Nichtform, des Seienden aus dem Nichtsein. Auf der Gegenseite haben wir die Kritik an der Rückführung auf das wahrhaft Seiende und die reine, identische Bestimmtheit. Was Metaphysik als das Wahre setzt

36 Ebd., S. 303 (dt.: S. 312).

37 J. Derrida, La voix et le phénomène, S. 75 (dt.: S. 92).

38 J. Derrida, „La différance“, S. 1-29 (S. 28 f.). 
und worin sie festen Halt sucht, wird von der Metaphysikkritik teils als labiles Fundament, teils als leeres Konstrukt, teils als Projektion eines falschen Bedürfnisses verworfen.

Nun ist diese Dialektik kein einfaches Wechselspiel von These und Antithese. Metaphysik ist in der Ursprungsfrage nicht einfach das Andere zum Mythos; umgekehrt ist die Kritik an ihr nicht eine Wiederherstellung der von ihr überwundenen Position. So werden auch in der späteren Ursprungskritik metaphysische und mythische Ansätze nicht notwendig als Differenz, sondern teils gerade in ihrer Gemeinsamkeit wahrgenommen (und etwa die ursprungsmythische Verhaftung der Metaphysik angeprangert). Gleichwohl ist es wichtig, die Divergenz beider Sichtweisen deutlich herauszustellen. Metaphysik unterscheidet sich darin vom Mythos, dass sie die dunkle Vorgeschichte der Form aus der Betrachtung ausschließt und verdrängt, während der Mythos die zu überwindende Negativität drastisch vorführt und in ritueller Wiederholung periodisch wiederbelebt. Im Mythos bleibt das Bewusstsein davon wach, dass Form und Identität nicht einfach - so die These der Metaphysik - suisuffizient, ja, das selbständig Seiende sind, sondern dass sie nur im Widerstand gegen die Zersetzung generiert werden und fortbestehen. In der Reminiszenz an einen tieferen Grund wird die Ursprungsreflexion zugleich der Brüchigkeit alles Seienden, der Bedrohtheit jeder Form und Ordnung gewahr. Vor diesem Hintergrund wird der Halt, den Metaphysik bei der festen Gestalt und beim Wesen sucht, in seiner Labilität durchschaut. Metaphysikkritik, die der Wahrheit des Mythos eingedenk ist, problematisiert die Prinzipien der Metaphysik als ungenügende Fundamente, aber auch als Gegenseite einer Ausblendung und Ausschließung: Die metaphysische arche gründet auf einem ,Ungedachten', das in einer vertieften Ursprungsbesinnung eingeholt und thematisiert werden muss. Damit ist gegen die Metaphysik eine radikalere, tiefer ansetzende Ursprungsreflexion verlangt.

Dies bedeutet, dass dort, wo Metaphysik und Mythos zum Gegenstand einer gemeinsamen Kritik am Ursprungsdenken werden wenn beispielsweise die Kritische Theorie die regressive Tendenz metaphysischer Ursprungsforschung mit der mythischen Vorherrschaft des Vergangenen zusammenbringt -, eine einseitige Wahrnehmung des Mythos bestimmend ist. Es ist eine Wahrnehmung, die in der mythischen Ursprungsbesinnung gerade nicht die negative Tiefenschicht, sondern die identitätsstiftende Ursetzung bzw. deren zyklische Wiederholung betont. Sie akzentuiert das Einswerden mit dem urzeitlichen 
Gründungsgeschehen, dem das gegenwärtige Leben seine Stabilität verdankt, wie die metaphysische Idee den an ihr teilhabenden Dingen ihr Wesen und ihr Sein verleiht. Unter Ausblendung der Differenz fokussiert solche Kritik auf das Gemeinsame von Mythos und Metyphysik - und jeder daran anschließenden Geisteshaltung in Historie, Tiefenpsychologie etc. -, das in der Suche nach dem Ersten, Ältesten und Ursprünglichen liegt. Sie wendet sich gegen die Vorherrschaft des Vergangenen, teils gegen die regressive Tendenz einer Vereinigung mit dem Anfang, und artikuliert den emanzipatorischen Widerstand gegen solche Festlegung auf das Anfängliche und Gewesene. Soweit diese gemeinsame Perspektive bestimmend bleibt, behält die Kritik ihre immanente Plausibilität. Zwischen dem Sichfestmachen an der identitätssichernden Herkunft und dem Eintauchen in die dionysisch-auflösende Vorwelt kann eine verwandte Tendenz bestehen, ein ähnliches Aufgehen im Ganzen und regredierendes Zurückgehen in den Grund. Der zukunftsgerichteten Geschichte sind der Mythos und die Metaphysik gleichermaßen fremd. Doch gerät die Kritik dort ins Unrecht, wo sie beide Positionen projizierend angleicht und die metaphysische arche im Zeichen des ewigen Kreislaufs deutet bzw. die ursprungsmythische Vergangenheit mit der Starre metaphysischer Identitätsfixierungen zusammenschließt, gegen welche (post-)modernes Denken das Eigenrecht der Pluralität und Differenz einklagt.

Insofern sind hier Präzisierungen erforderlich, die gleichzeitig eine bestimmte Rehabilitierung des Ursprungsdenkens einschließen. Genauer geht es um eine zweifache Rehabilitierung des Ursprungsdenkens, die schematisch den beiden Typen des Mythos (4.2) und der Metaphysik (4.3) zuzuordnen ist.

\subsection{Mythischer Ursprung und Negativität}

Auf der einen Seite ist an dem festzuhalten, worin die mythische über die metaphysische Ursprungsbesinnung hinausweist (bzw. diese gegenüber jener eine Verkürzung, einen Verlust darstellt): im Sichtbarmachen der Negativität des Urgrundes (gegenüber dem affirmativen Fundament der Metaphysik) und im Geltendmachen von Pluralität und Differenz (gegenüber der Zentrierung auf das Einheitlich-Identische).

Der erste Aspekt, auf dem im Vorigen der Hauptakzent lag, weist auf ein aufklärerisches, emanzipatorisches Potential hin, dessen sich eine kritische Ursprungsreflexion versichern muss. Es geht nicht nur darum, ein Verdrängtes reflexiv einzuholen, sondern sich der Negativität, dem 
Leiden und der Bedrohtheit auszusetzen, um sich aus dieser Erfahrung und diesem Bewusstsein heraus über Wirklichkeit zu verständigen. Um sich erinnernd eigener oder fremder Identität zu vergewissern, ist es wichtig, nicht nur den Anfang einer Geschichte, den affirmativen Stiftungs- oder Gründungsakt zu vergegenwärtigen, sondern daran zu erinnern, wogegen sich diese Gründung behaupten musste, wovon sie sich abgelöst und was sie überformt hat.

Der zweite Aspekt ist derjenige, den vor allem ein nachmetaphysisches Denken gegen die metaphysischen Festlegungen - gegen die großen Erzählungen, die Unterdrückung von Vielfalt und Wandel geltend macht. Auch hier können wir sagen, dass sich das ältere, von der Metaphysik überwundene Ursprungsdenken in einem weiteren Horizont bewegt und die Buntheit und Ungeordnetheit der Lebenswelt zum Tragen bringt. Es bringt zur Geltung, was noch nicht der Vereinheitlichung, Purifizierung und Festschreibung durch metaphysisch-rationale Prinzipien unterworfen ist. Allerdings dürfen wir die Annäherung zwischen vormetaphysischen und nachmetaphysischen Gegenpositionen zur Metaphysik nicht vorschnell vollziehen (auch wenn sich im Horizont der Letzteren Ansätze einer Neuaneignung des Mythos finden). Was der Identität vorausliegt und durch sie überformt wird und was aus ihr entlassen, ,später ${ }^{6}$ als sie ist, ist nicht von derselben Art: Die durch die Kosmosbildung überwundene Ordnungslosigkeit und Leere steht für ein Negatives und Bedrohliches, während die aus der Einheit entlassene Vielfalt und Veränderlichkeit im Zeichen der Indifferenz und Offenheit erscheint. Das Zurückgehen hinter die Bildung von Ordnung und Gestalt und das Sicheinrichten im Abgeleiteten und Relativen, der undurchdringliche Abgrund und die Spiegelung der Oberfläche sind entgegengesetzte Antithesen zum Wesen. Insofern ist die Metakritik nicht Rückkehr zum Ersten, Metaphysikritik nicht Rückgang zum Mythos. Darin liegt nicht nur eine Korrektur an einer bestimmten (Selbst-)Wahrnehmung nachmetaphysischer Ursprungskritik. Darin liegt auch eine bestimmte Metakritik an der Letzteren, damit eine bestimmte Rehabilitierung der von ihr zurïckgewiesenen metaphysischen Ursprungsorientierung.

\subsection{Metaphysischer Ursprung und die Unhintergehbarkeit des Ersten}

Rehabilitierung der metaphysischen Ursprungsorientierung meint ein Festhalten an ihrer Frage gegen deren Suspendierung im modernen Funktionalismus oder postmodernen Indifferentismus. Tatsache ist, dass 
die vielfach beanspruchte Einsicht in die Ungesichertheit der metaphysischen Fundamente die Frage nach dem Ersten nicht abgeschafft, das Bedürfnis nach einer Orientierung am Ursprung nicht zum Verschwinden gebracht hat. Bei aller Kritik am Konstruktcharakter der substantiellen Form, bei aller Abwehr der regressiv-destruktiven Funktion der Ursprungsversenkung bleibt die identitätsstiftende Kraft zu explizieren, die den Anfangs- und Ursprungsbildern unabhängig von ihrem Wahrheitsgehalt innewohnt und deren das individuelle wie soziale Leben bedarf; trotz aller epistemologischen Fragwürdigkeit letzter Prinzipien ist die von Aristoteles behauptete Nötigung, nach ersten und absoluten Gründen zu suchen, nicht suspendiert. Es liegt nahe, die Suche nach letzten Gründen in der Reihe jener metaphysischen Orientierungen zu sehen, die das menschliche Denken nach Kant nicht positiv einholen, doch ebensowenig gleichgültig verabschieden kann. Nicht die Hypostasierung des Ersten, sondern seine umstandslose Preisgabe wird in solcher Sicht zur leeren Setzung. Die fundamentalistische Beschwörung des Ursprünglichen und die Leichtigkeit des vom Grunde Abgelösten sind gleichermaßen , unbewiesene', ungesicherte Haltungen. Die Frage nach dem Ursprung ist keine kontingente Ausrichtung menschlicher Vernunft, auch wenn uns ihre letztgültige Antwort versagt bleibt und ihre vergegenständlichende Festlegung ihrerseits zur Bedrohung und zerstörenden Regression werden kann.

Am konsequentesten scheinen dem jene Ursprungsfiguren Rechnung zu tragen, die sowohl an der Intransigenz der Suche nach dem Ersten wie an dessen Nicht-Einholbarkeit festhalten. Explizit hat sich die Dekonstruktion über eine ,Subversion“ metaphysischer Gegensätze definiert, die nicht einfach die gängigen Hierarchien (Grund-Begründetes, Einheit-Vielfalt, Identität-Differenz) umkehren, sondern sie in ihrem Kern auflösen und neu fassen will. Nicht etwas anderes soll anstelle der Substanz oder der Urstiftung als wahres Fundament statuiert, sondern die Idee des bestimmten, identifizierbaren Grundes soll selbst problematisiert werden - ohne sie als Frage zu disqualifizieren oder ihr erkenntnisleitendes Interesse einfach zu widerrufen. ,Urspur ${ }^{6}$ und ,différance sind Chiffren dieser zwiespältigen Aufhebung, welche das Anliegen der Metaphysik in seiner klassischen Gestalt unterläuft, indem sie es in gewissem Sinne zugleich radikalisiert und seine Uneinlösbarkeit denkt. In vielfachen Umschreibungen hat J. Derrida diesem abgründigen Zwiespalt, der ein Zwiespalt im Wahrheitsanspruch des Philosophierens ist, Ausdruck verliehen. In vielleicht noch eindringlicheren Formulierungen hat Th. W. Adorno den vergleichbaren Zwiespalt 
negativer Dialektik gefasst, die sich einem Anspruch des Denkens unterstellt, den sie als ebenso unhintergehbar wie unerfuillbar beschreibt so im Schlusspassus der Negativen Dialektik, der das geforderte Denken als „solidarisch mit der Metaphysik im Augenblick ihres Sturzes“ erklärt. ${ }^{39}$

Bedeutsam sind die - gänzlich verschiedenen und doch in der Tiefe verwandten - Denkfiguren Derridas und Adornos in unserem Kontext deshalb, weil sie eine Art Schlussfigur in der Ursprungskonstellation zu zeichnen erlauben. Die von Adorno evozierte ,Solidarität' mit der Metaphysik im Augenblick ihres Sturzes verweist auf eine Rettung des metaphysischen Motivs, in welcher gleichzeitig die Negativität der von der Metaphysik verdrängten Tiefenschicht fassbar wird. Adorno und Derrida sind ,negativistische' Denker, die gegen die Affirmativität metaphysischer Spekulation den Ausgang vom Negativen - vom Leiden, vom Sinndefizit, von realer Falschheit - als Grund und Impuls des Gedankens setzen. Sie widersprechen der Geschlossenheit der Welt und der Wahrheit ihrer Fundamente, ohne ihnen das Grundlose zu substituieren und beim Beliebigen Zuflucht zu suchen. In der Linie dieses Denkens ließe sich eine Restitution des Ursprungsdenkens ins Auge fassen, die auf einen Ursprung ohne Substantialisierung hin denkt und die Identität des Ersten als grundsätzlich infrage gestellte wahrnimmt. Das problematisierte, seiner nicht sichere Erste ist darin der tiefste Ursprung. Es wäre ein Ursprungsdenken, in welchem die Unnachgiebigkeit der Suche nach dem radikal Ersten und Voraussetzungslosen sich mit dem Bewusstsein der Abgründigkeit des Negativen, der Bedrohtheit jeder positiven Setzung und der Brüchigkeit jedes Anfangs verbindet. In einen Begriff zusammengeführt, könnte man diese Verflechtung der ,metaphysischen' mit der ,mythischen' Stoßrichtung der Ursprungsreflexion im Konzept einer ,negativen Metaphysik' fassen. Doch geht es nicht nur um die Übernahme und Verbindung überlieferter Ansätze. Ebenso wichtig ist die Distanzierung von ihnen, gerade in dem, was ihre gemeinsame Vergangenheitsorientierung ausmacht. Es wäre ein Ursprungsdenken, das sich sowohl von der affirmativ-metaphysischen Prinzipienlehre wie von der überhöhenden Figur des unvordenklichen Einen ablöst, das sich aber ebenso - trotz des gemeinsamen negativistischen Ansatzes - aus dem Bann der mythischen Wiederkehr befreit: Die Suche gilt einem Ursprung, der nicht die Herrschaft des Vergan-

39 Th. W. Adorno, Negative Dialektik, Frankfurt am Main 1967, S. 398; vgl. den Schlussaphorismus der Minima Moralia, Frankfurt am Main 1969. 
genen perpetuiert und sein Anderes in sich einbehält, sondern es in eine offene Zukunft hinein freigibt. Es wäre ein Ursprung, der ebensosehr Anfang ist.

\subsection{Die Ambivalenz des Ursprungsdenkens}

Man kann sich fragen, wieweit es gelingt, im Zusammenbringen der gegenläufigen Impulse am Ende eine Figur des Ursprungsbezugs zu zeichnen. Unverkennbar ist es eine Figur, die ein Spannungsverhältnis in sich birgt, eine ungelöste Spannung zweier divergierender, gegenstrebiger Richtungen der Ursprungssuche umgreift. Es sind zwei Stoßrichtungen, die beide in der Ideengeschichte in prägnanter, eindringlicher Gestalt auftreten, als Äußerungen eines Strebens nach Einholung des Ursprungs, zum Teil als Ausdruck einer originären Ursprungssehnsucht, wie sie in Anthropologie und Religionswissenschaft beschrieben wird.

Das eine Streben ist dasjenige, das immer tiefer gehen, hinter die Welt und jeden bestimmten Anfang zurückgehen will, der immer ein Anfang innerhalb eines Umfassenderen, nie der absolute, aus sich selbst kommende Anfang ist. Es ist die Sehnsucht, in den tiefsten Ursprung zurückzugehen, mit dem Urgrund eins zu werden, der hinter jeder bestimmten Gestalt und jedem konkreten Beginn zurückliegt. In der Fluchtlinie dieses Strebens liegt die Sehnsucht, Grenzen zu überschreiten und im Ganzen aufzugehen, sich im Grenzenlosen aufzulösen. Es ist eine Sehnsucht, die ihrerseits, wie Nietzsche am Beispiel des Dionysischen gezeigt hat, durch eine tiefste Ambivalenz gezeichnet ist, sofern das Aufgehen im Ganzen als höhere Selbstwerdung wie als radikaler Selbstverlust, als höchste Erfüllung wie als Schrecken erfahren werden kann. Auf der Gegenseite steht das Streben, eine letzte Bestimmtheit als sicheren Halt und festen Identitätsgrund $z u$ finden, das Streben nach Rückkehr in die Heimat und die bestimmte Herkunft. Nicht Auflösung im Urgrund, sondern Selbstfindung im Anfang ist hier das Erstrebte. Der Schrecken vor dem Unendlichen, den Aristoteles als Kehrseite der Suche nach dem Ersten evoziert, geht in die Motivationsbasis dieses Strebens ebenso ein wie das positive Bedürfnis nach Selbstaffirmation und Identität.

Die Frage ist, wieweit wir hier mit einer oder mit zwei Formen der Suche nach dem Ursprung zu tun haben. Es scheint unstrittig, dass wir im Konkreten in den meisten Fällen den einen oder den anderen Weg verfolgen, die eine oder die andere Weise, zum Ersten zurück- und vom Ersten auszugehen, praktizieren. In der historischen Erforschung einer 
kulturellen Neuerung können wir von Gründungsinitiativen oder von deren offener Vorgeschichte ausgehen, wir können von dem einen oder dem anderen ,Ursprung' her Aufschluss darüber suchen, wie diese Neuerung zustande gekommen ist und worum es in ihr geht; ähnlich kann die wissenschaftliche Erklärung eines Naturphänomens auf die unmittelbar auslösenden Faktoren oder auf die langfristigen und globalen Prozesse, die ihnen zugrundeliegen, zurückgreifen, wobei die Triftigkeit der einen oder anderen Perspektive von Zweck und Themenstellung der Erklärung abhängig ist. Gleichwohl zögern wir, die Frage nach dem Ursprung, wo wir in einem emphatischen Sinn mit ihr zu tun haben, in diese Doppelgestaltigkeit zu entlassen. Es scheint ein Anliegen, ein Interesse zu sein, dem wir in Religionen, Weltbildern und philosophischen Spekulationen begegnen, auch wenn es sich als doppelgesichtig, zwiefältig erweist. Die beiden Fluchtlinien streben nicht nur auseinander, sondern nehmen aufeinander Bezug, weisen vom einen Gravitationspunkt zum anderen. Wenn wir in der Ursachenkette zum letzten Glied zurückgehen, nach dem irreduziblen Anfang oder dem tiefsten Grund forschen, so kann dieses Prinzip einem zweiten Blick als eines erscheinen, das nicht in Selbstevidenz gegeben ist, sondern selbst in Frage steht, über sich hinaus weist, nach einem Grund seiner selbst verlangt. Die reklamierte Apodiktizität, die dem Ersten als Gegenstand einer ursprünglichen Intuition oder eines rationalen $\mathrm{Be}-$ weises zukommen soll, kann sich als brüchig und hohl herausstellen; die Unerschütterlichkeit des Fundaments, auf dem alles gründen soll, kann sich als labil erweisen. Wenn das Abzielen auf letzte Prinzipien die Angst vor dem Chaos bannen soll, so verbürgt die Intention nicht ihre Erfüllung. Der feste Grund kann den Blick in den Abgrund freigeben. Die Suche nach dem bestimmten Anfang kann sich in die Erforschung der Vorgeschichte, der offenen Herkunft hinein fortsetzen; die Sehnsucht nach dem Ursprung kann sich über den Anfang hinaus auf den Urgrund, das Unendliche ausweiten. Schließlich wohnt dem Urgrund selbst die Ambivalenz zwischen tragendem Fundament und verschlingendem Abgrund inne: Das Zurückgehen in ihn kann als Aufgehobensein und Getragenwerden, aber auch als Absorbiertsein und Auflösung erlebt werden. Was in den Ursprung zurückgeht, kann sich in diesem finden oder verlieren.

Solche schillernden Übergangsphänomene setzen nicht die Distinktheit der Motive und Fragerichtungen außer Kraft. Die Suche nach dem Ursprung artikuliert sich in gegenläufigen Strebungen, die nicht auf eine Intention, eine Sehnsucht, eine Frage reduzierbar sind. Sie sind in 
dieser Spannung beizubehalten und gleichzeitig in ihrer Affinität zu reflektieren. In dieser Zwiefältigkeit, in ihrer Ambivalenz ist die Frage nach dem Ursprung eine Frage, die der menschlichen Verständigung wesentlich ist und an der gegen ihre Kritik und Obsoleterklärung festzuhalten ist. 